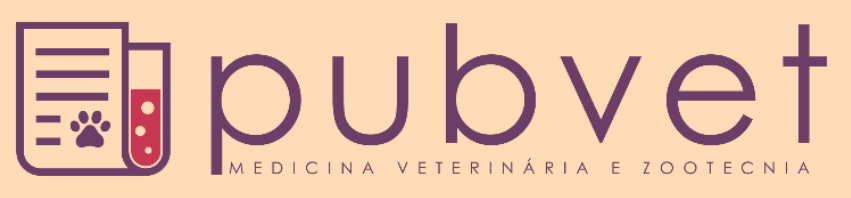

https://doi.org/10.31533/pubvet.v15n03a763.1-10

\title{
Comportamento de vacas mestiças em pastejo de capim 'Mombaça' e características de forragem no semiárido
} \author{
Dinamérico de Alencar Santos Júnior ${ }^{20}$ \\ ${ }^{I}$ Estudante de Medicina Veterinária da Universidade Federal do Oeste da Bahia (Barra, Bahia, Brasil). \\ ${ }^{2}$ Professor(a) da Universidade Federal do Oeste da Bahia, Centro Multidisciplinar de Barra. Barra-BA Brasil. \\ *Autor para correspondência, E-mail: janaina.lima@ufob.edu.br
}

Monica dos Santos de Mello ${ }^{\bullet}$, Rodrigo Gonçalves de Carvalho ${ }^{1 \bullet}$, Jordana Cunha $^{1 \bullet}$, Janaina de Lima Silva ${ }^{2 *} \bullet$, Caio Victor Damasceno Carvalho ${ }^{2} \bullet$, Adérico Júnior Badaró Pimentel ${ }^{2} \bullet$,

Resumo. Objetivou-se avaliar quatro intensidades de pastejo $(10,25,40$ e $55 \mathrm{~cm}$ de altura de resíduo) sobre as características produtivas e comportamento ingestivo de vacas mestiças sob pastejo em Panicum maximum Jacq. 'Mombaça', no semiárido baiano. O delineamento utilizado no experimento foi o Inteiramente Casualizado (DIC), sendo quatro tratamentos (intensidade de pastejo) e cinco repetições (piquetes). Utilizaram-se cinco vacas mestiças (Holandês x Gir), com peso médio de $500 \mathrm{~kg}$, como animais avaliadores. As avaliações do pasto foram realizadas por meio de pastejo simulado a cada 28 dias. $\mathrm{O}$ comportamento ingestivo foi avaliado por meio de observação visual e a temperatura da superfície de pelame foi aferida com termômetro digital após a ordenha e durante o pastejo dos animais. Verificou-se aumento linear $(\mathrm{P}<0,05)$ para a massa seca total $(\mathrm{MST})$, massa seca de lâminas (MSL), taxa de acúmulo de forragem (TXAF), oferta de massa seca total (OMST) e oferta de massa seca de lâminas (OMSL). A porcentagem mínima de lâminas foliares $(\mathrm{P}<0,05)$ de $82,2 \%$ foi observada na altura de resíduo de $34,9 \mathrm{~cm}$. Observou-se que o Índice de Temperatura e Umidade (ITU) ficou em atenção e alerta durante os meses de avaliação. Verificou-se aumento linear $(\mathrm{P}<0,05)$ da temperatura da superfície de pelame de 32,2 para $36,4^{\circ} \mathrm{C}$, ao longo dos meses de avaliação. Para cada incremento de $5,0 \mathrm{~cm}$ na altura de resíduo, espera-se um aumento $(\mathrm{P}<0,05)$ de $0,146 \mathrm{n} \%$ minuto e $52,6 \mathrm{n} \%$ dia na taxa de bocados, respectivamente. Para a estação alimentar, espera-se uma redução $(\mathrm{P}<0,05)$ de $0,162 \mathrm{n} \%$ minuto e $58,2 \mathrm{n} \%$ dia, respectivamente. O consumo de matéria seca (CMS) e ganho médio diário $(\mathrm{GMD})$ aumentaram linearmente $(\mathrm{P}<0,05)$ entre as intensidades de pastejo. Nas intensidades de pastejo de 40 e $55 \mathrm{~cm}$ houve uma variação de 3,25 para 3,50 no escore de condição corporal (ECC). Recomenda-se a intensidade de pastejo de $45,2 \mathrm{~cm}$ (altura de resíduo), por permitir adequada produção de forragem e de bocados, e menor deslocamento entre estações alimentares.

Palavras chave: estação alimentar, manejo de pastagem, oferta de forragem, Panicum maximum

\section{Behavior of crossbred cows grazing 'Mombaça' grass and forage characteristics in the semiarid region}

Abstract. The objective was to evaluate four grazing intensities (10, 25, 40, and $55 \mathrm{~cm}$ of residue height) on the yield characteristics of Panicum maximum Jacq. 'Mombaça' and feeding behavior of crossbred cows under grazing in the Bahian semiarid. The design used in the experiments was a Completely Randomized Design (CRD), with four treatments (grazing intensity) and five repetitions (paddocks). Five crossbred cows (Holstein x Gir) with an average weight of $500 \mathrm{~kg}$ were used as evaluator animals. Pasture evaluations were carried out through simulated grazing every 28 days. Ingestive behavior was assessed by 
visual observation and the temperature of the corporal surface was measured with a digital thermometer after milking and during the grazing period of the animals. There was a linear increase $(\mathrm{P}<0.05)$ for total dry mass (TDM), leaves dry mass (LDM), forage accumulation rate (FAR), total dry mass supply (TDMS), and leaves dry mass supply (LDMS). The minimum percentage of leaves $(\mathrm{P}<0.05)$ of $82.2 \%$ was observed at the residue height of $34.9 \mathrm{~cm}$. It was observed that the Temperature and Humidity Index (ITU) was in attention and alert during the months of evaluation. There was a linear increase $(\mathrm{P}<0.05)$ in the temperature of the corporal surface from 32.2 to $36.4{ }^{\circ} \mathrm{C}$, over the months of evaluation. For each increase of $5.0 \mathrm{~cm}$ in the residue height, an increase $(\mathrm{P}<0.05)$ of $0.146 \mathrm{n} /$ minute and $52.6 \mathrm{n} \%$ day is expected in the bit rate, respectively. For the feeding station, a reduction $(\mathrm{P}<0.05)$ of $0.162 \mathrm{n} \%$ minute and $58.2 \mathrm{n} \%$ day is expected, respectively. The dry matter intake $(\mathrm{DMI})$ and average daily gain $(\mathrm{ADG})$ increased linearly $(\mathrm{P}<0.05)$ between grazing intensities. At the grazing intensities of 40 and $55 \mathrm{~cm}$, there was a variation from 3.25 to 3.50 in the body condition score (BCE). Grazing intensity of $45.2 \mathrm{~cm}$ (residue height) is recommended, as it allows an adequate forage yield and bites, and less displacement between feeding stations.

Keywords: feeding station, forage supply, Panicum maximum, pasture management

\section{Introdução}

A pecuária no Brasil é desenvolvida basicamente em pastagens tropicais (Rocha et al., 2019) onde o manejo e utilização da forragem representam grande parte da eficiência do sistema (Morais et al., 2018). O manejo de pastagens pode ser avaliado pela intensidade do pastejo e esta é influenciada pela espécie forrageira, pois cultivares distintas apresentam diferenças na morfologia, composição e produtividade. Também, o comportamento ingestivo dos ruminantes a pasto pode ser influenciado pela estrutura do dossel, caracterizada pela altura, relação folha/colmo, densidade de forragem e massa de lâmina foliar (Souza et al., 2011), além de fatores abióticos, como a temperatura ambiente (Sollenberger \& Cherney, 1995).

Flores et al. (2008) destacaram que o monitoramento e o controle da altura do dossel geram relações bastante consistentes entre a resposta da forrageira e o desempenho dos animais à pasto. Carnevalli et al. (2006) ao avaliarem diferentes intensidades de pastejo em pastagem de capim 'Mombaça' verificaram alto acúmulo de matéria seca com altura pós-pastejo de $30 \mathrm{~cm}$. Reis et al. (2009) observaram que em diversos estudos houve incremento no ganho de peso dos animais com a elevação na quantidade e qualidade de forragem ofertada.

A avaliação do comportamento ingestivo associado aos parâmetros fisiológicos pode contribuir para a adequação do manejo dos animais à pasto (Silva et al., 2009). Souza et al. (2011) constataram que em pastagens de milheto e papuã, manejadas a $40 \mathrm{~cm}$ de altura de dossel, não houve efeitos sobre o comportamento ingestivo de novilhas, medido pela mudança de estações alimentares e deslocamentos. Para o semiárido brasileiro são escassos os estudos envolvendo o manejo do pastejo e o comportamento ingestivo de bovinos de leite. Assim, objetivou-se com esse trabalho avaliar o efeito da intensidade de pastejo sobre características produtivas do capim 'Mombaça' e o comportamento ingestivo de vacas mestiças, de aptidão leiteira, no semiárido baiano.

\section{Material e métodos}

O estudo foi realizado no Sítio Curralinho, localizado no município de Barra, Bahia, em pastagem de Panicum maximum Jacq. 'Mombaça', no período de agosto a dezembro de 2019. O município de Barra localiza-se na região semiárida do Oeste da Bahia, a aproximadamente $406 \mathrm{~m}$ de altitude, $11^{\circ} 05^{\prime} 20^{\prime}$ 'S de latitude e $43^{\circ} 08^{\prime} 31^{\prime}$ 'O, sendo o tipo climático caracterizado como BSh (semiárido quente), segundo classificação de Köppen, com temperatura média anual de $25,7^{\circ} \mathrm{C}$ e pluviosidade média anual de $649 \mathrm{~mm}$.

Os tratamentos avaliados foram quatro intensidades de pastejo: 10, 25, 40 e $55 \mathrm{~cm}$ de altura de resíduo. Estes foram dispostos segundo o Delineamento Inteiramente Casualizado com cinco repetições (piquetes). A área utilizada para pastejo no experimento foi de 1,20 ha, composta de 20 piquetes de 0,06 hectares $(20$ x $30 \mathrm{~m})$. O método de pastejo utilizado foi o de lotação rotativa com taxa de lotação fixa. 
Cada piquete foi pastejado por cinco vacas mestiças (animais avaliadores) de aptidão leiteira, em final de lactação, com peso corporal (PC) médio de $500 \mathrm{~kg}$. Todos os procedimentos experimentais foram aprovados pela Comissão de Ética no Uso de Animais da Universidade Federal do Oeste da Bahia, protocolo n. 0020/2019.

A altura de resíduo da forragem foi medida mensalmente, utilizando-se uma trena, graduada em centímetros. Foram medidos cinco pontos aleatórios por piquete, sendo a altura em cada ponto correspondente à altura média da curvatura das folhas superiores em torno da trena. A coleta de pasto foi efetuada segundo Sollenberger \& Cherney (1995), por meio de simulação de pastejo. As amostras foram coletadas pelo método hand-plucking, no qual coleta-se manualmente a forragem depois de acurada observação do hábito de pastejo dos animais. Foram coletados aproximadamente $300 \mathrm{~g}$ de forragem fresca por unidade experimental, uma vez ao mês, durante os períodos de coleta de todo o período experimental. As amostras compostas foram separadas manualmente em lâmina foliar, colmo (colmo e bainha) e material morto.

A massa de forragem foi estimada a cada 28 dias cortando-se seis amostras coletadas em área de 1 $\mathrm{m}^{2}$, rente ao solo, alocadas ao acaso, segundo metodologia utilizada por Flores et al. (2008). As amostras de lâminas foliares, colmos e material morto foram secas em estufa de ventilação forçada a $60{ }^{\circ} \mathrm{C}$ por 72 horas e pesada para estimativa da massa seca (Método INCT-CA G-001/1), de acordo com metodologia descrita por Detmann et al. (2012). O acúmulo de forragem $(\mathrm{kg} / \mathrm{ha}$ de massa seca) foi obtido pela aferição das massas de forragem registradas no piquete a cada avaliação mensal. Para a estimativa da taxa de acúmulo de forragem $(\mathrm{kg} / \mathrm{ha} / \mathrm{dia})$, foi dividido o acúmulo de forragem por 28 , equivalente ao número de dias de descanso do piquete. A oferta de matéria seca ( $\mathrm{kg}$ de MS por $100 \mathrm{~kg}$ de PC) foi calculada utilizando-se a soma da massa seca presente no piquete e o acúmulo de forragem no período dividida pelo total do peso corporal (PC) mantido no piquete no mesmo período (Flores et al., 2008). A oferta de lâmina foliar ( $\mathrm{kg}$ de massa seca de lâmina foliar por $100 \mathrm{~kg}$ de PC) foi calculada da mesma forma, considerando apenas esse componente da planta.

Após o manejo no curral, as vacas foram levadas para a área de pastagem, onde permaneceram das 7:00 $\mathrm{h}$ às 15:00 $\mathrm{h}$ para pastejo. As avaliações do comportamento ingestivo foram realizadas por meio de observação visual, com intervalos de dez minutos, segundo metodologia de Jamieson \& Hodgson (1979). Os animais avaliadores foram observados durante todo o período de pastejo e suas atividades de mastigação (bocados) e deslocamento nas estações alimentares foram contabilizadas, conforme metodologia adaptada de Flores et al. (2008) e Palhano et al. (2006). Uma estação alimentar foi considerada como o semicírculo hipotético disponível em frente ao animal que pode ser alcançado sem que seja necessário mover as patas dianteiras. $\mathrm{O}$ número de bocados por estação foi calculado pela divisão entre o número diário de bocados e o número diário de estações alimentares. $\mathrm{O}$ número de estações por minuto foi calculado pela divisão do número diário de estações pelo tempo de pastejo. Essas observações foram realizadas por cinco observadores, sendo um observador por animal.

Os parâmetros fisiológicos foram avaliados de acordo com metodologia utilizada por Andrade et al. (2017). A temperatura da superfície de pelame (TSP) foi aferida durante o período de coleta de dados, no momento após as ordenhas e durante o período de pastejo dos animais, utilizando-se termômetro digital com infravermelho, o qual foi posicionado a cerca de um metro de distância dos animais. Foram realizadas três aferições de temperatura por animal, para posterior registro da média de temperatura de pelame por animal e por tratamento.

O peso corporal das vacas foi estimado a cada 30 dias, por meio de medição $(\mathrm{cm})$ do perímetro torácico (PT), utilizando-se fita comercial Suprivet ${ }^{\circledR}$. O escore da condição corporal (ECC) foi estimado nos dias de pesagem dos animais, com base em avaliações visuais e táteis das reservas corporais em pontos específicos do corpo do animal, utilizando-se uma escala biológica, atribuindo-se valores que variaram de 1 a 5 , em que $1=$ muito magro e $5=$ muito gordo, com intervalo de 0,25 pontos, de acordo com o critério proposto por Ferguson et al. (1994). O Vetscore ${ }^{\circledR}$ foi utilizado para classificar o ECC em três status: 1) vermelho, vacas com $\mathrm{ECC}<2,75$; 2) verde, vacas com 2,75 $\leq \mathrm{ECC} \leq 4,5$; e 3) amarelo, vacas com ECC > 4,5, de acordo com Pfeifer et al. (2017).

Os dados coletados foram submetidos à análise de variância (ANOVA) e regressão, ao nível de significância de 5\%, utilizando-se o sistema de análises estatísticas do software SAS. 


\section{Resultados e discussão}

Ao longo do estudo, a altura de dossel no pré-pastejo variou de 30,5 a 90,4 cm, e no pós-pastejo o resíduo variou de 10,2 a 55,4 $\mathrm{cm}$ (Tabela 1). Carvalho et al. (2017) ao avaliarem pastos de capim 'Mombaça' registraram alturas médias de pré-pastejo de 91,0 e 92,0 cm para os tratamentos com 30 e $50 \mathrm{~cm}$ de resíduo pós-pastejo, respectivamente. Os resultados do presente estudo indicaram que a altura de pasto recomendada para a entrada de animais (pré-pastejo) seria de 90,4 cm, enquanto o alvo de altura residual foi atingido na condição pós-pastejo de 40,1 cm, mas na altura de pré-pastejo de 78,0 cm.

Tabela 1. Alturas, massas e porcentagem de forragem de capim 'Mombaça' manejado sob diferentes intensidades de pastejo no semiárido baiano.

\begin{tabular}{|c|c|c|c|c|c|c|c|}
\hline \multirow{2}{*}{ Variáveis } & \multicolumn{4}{|c|}{ Altura de resíduo, $\mathrm{cm}$} & \multirow{2}{*}{ EPM* } & \multicolumn{2}{|c|}{$\mathrm{P}>$ Valor } \\
\hline & 10 & 25 & 40 & 55 & & Linear & Quadrático \\
\hline \multicolumn{8}{|c|}{ Alturas observadas, $\mathrm{cm}$} \\
\hline Pré-pastejo & 30,5 & 55,0 & 78,0 & 90,4 & - & - & - \\
\hline Pós-pastejo & 10,2 & 25,3 & 40,1 & 55,4 & - & - & - \\
\hline \multicolumn{8}{|c|}{ Massa seca, kg/ha } \\
\hline Total & 2.475 & 4.484 & 9.087 & 13.512 & 161 & $0,000^{\mathrm{a}}$ & 0,000 \\
\hline Lâminas & 2.311 & 4.001 & 7.064 & 12.446 & 219 & $0,000^{\mathrm{b}}$ & 0,000 \\
\hline Colmos & 96,3 & 318 & 1.222 & 761 & 55,6 & 0,000 & $0,000^{c}$ \\
\hline Material morto & 47,1 & 165 & 800 & 305 & 44,9 & 0,012 & $0,001^{\mathrm{d}}$ \\
\hline \multicolumn{8}{|c|}{ Porcentagem, $\%$} \\
\hline Lâminas foliares & 93,4 & 89,2 & 77,7 & 92,1 & 0,930 & 0,242 & $0,000^{\mathrm{e}}$ \\
\hline Colmo & 3,89 & 7,09 & 13,4 & 5,64 & 0,470 & 0,120 & $0,000^{\mathrm{f}}$ \\
\hline Material Morto & 1,90 & 3,69 & 8,81 & 2,25 & 0,420 & 0,295 & $0,000^{\mathrm{g}}$ \\
\hline
\end{tabular}

*Erro padrão da média. ${ }^{a} \mathrm{y}=782,33+251,43 \mathrm{x}$; ${ }^{\mathrm{b}} \mathrm{y}=-795,92+223,12 \mathrm{x} ;{ }^{\mathrm{c}} \mathrm{y}=-616,48+68,627 \mathrm{x}-0,7586 \mathrm{x}^{2}$; $\mathrm{d}=-503,72+$

$53,656 x-0,681 x^{2} ;{ }^{e} y=107,45-1,446 x+0,0207 x^{2} ;$ f $y=-4,4374+0,8686 x-0,0122 x^{2} ;{ }^{g} y=-4,3646+0,6442 x-0,0093 x^{2}$.

Segundo Carnevalli et al. (2006), a altura do pasto é um parâmetro prático e confiável para uso como guia ou referência de campo para monitorar e gerenciar o manejo de pastagem. As intensidades de pastejo recomendadas para manejo de capim 'Mombaça' em sistema de lotação rotativa variam entre 30 e $50 \mathrm{~cm}$, de acordo com Silva et al. (2009). Estas alturas para o resíduo de forragem pós-pastejo são necessárias para que as plantas forrageiras possam ter reservas suficientes para se recuperarem e rebrotar rapidamente. Além disso, sob pastejo rotativo, o período de ocupação e as atividades de pastejo estão intimamente associadas com a altura residual de folhas, ou seja, as características estruturais do pasto estão associadas à eficiência de pastejo, independente do consumo diário de forragem (Ribeiro Filho et al., 2011).

Verificou-se aumento linear $(\mathrm{P}<0,05)$ nas massas secas $(\mathrm{MS})$ totais e de lâminas, ou seja, para cada aumento de $5,0 \mathrm{~cm}$ na altura de resíduo, espera-se um aumento de 1.257 e $1.116 \mathrm{~kg} / \mathrm{ha}$ de MS total e de lâminas, respectivamente (Tabela 1). Comportamento quadrático $(\mathrm{P}<0,05)$ foi observado nas MS de colmos $(936 \mathrm{~kg} / \mathrm{ha})$ e de material morto $(553 \mathrm{~kg} / \mathrm{ha})$, com produção máxima nas alturas de resíduo de 45,2 e 39,4 cm, respectivamente (Tabela 1). Segundo Reis et al. (2009), a maximização da produção forrageira deve ser baseada no ajuste da intensidade de pastejo, levando-se em consideração a massa de forragem disponível para o animal, para se controlar a qualidade e a quantidade de forragem. Em todas as intensidades de pastejo estudadas a quantidade de MS total (Tabela 1) foi sempre superior ao limite de $2.000 \mathrm{~kg} / \mathrm{ha}$, sugerido por Minson (2012). Este parâmetro é considerado o mínimo de forragem disponível em pastagem de gramíneas tropicais para não se restringir o consumo de forragem pelos animais. A maior produção de MS de lâminas na intensidade de pastejo de 55,0 cm era prevista, uma vez que, os animais tinham maior quantidade de forragem disponível em maior altura de dossel. Segundo Flores et al. (2008), esse resultado se deve ao fato de os animais não precisarem explorar os estratos inferiores, deixando acumular maior quantidade de tecido foliar.

Para a porcentagem de lâminas foliares verificou-se um valor mínimo $(\mathrm{P}<0,05)$ de $82,2 \%$, na altura de resíduo de 34,9 cm, e porcentagens máximas $(\mathrm{P}<0,05)$ de colmos e material morto de 11,0 e 6,8\%, nas alturas de resíduos de 35,6 e 34,6 cm, respectivamente (Tabela 1). Carvalho et al. (2017) ao avaliarem diferentes estratégias de manejo em pastos de capim 'Mombaça', observaram maior proporção de caules e material morto para o resíduo pós-pastejo de $30 \mathrm{~cm}$, o que foi resultante da maior intensidade de desfolha e seletividade pelos animais em pastejo. $\mathrm{O}$ manejo da altura do pasto é uma importante ferramenta para se controlar a produção de colmo, uma vez que mais colmo e material morto 
em relação à folha, prejudicaria o desempenho e a produtividade dos animais em pastejo (Alexandrino et al., 2011; Carnevalli et al., 2006).

A taxa de acúmulo de forragem (TXAF), oferta de massa seca total (OFMST) e oferta de massa seca de lâminas foliares (OFMSL) aumentaram linearmente ( $\mathrm{P}<0,05)$, ou seja, para cada aumento de $5,0 \mathrm{~cm}$ na altura do resíduo, espera-se um aumento de 41,8 kg MS/ha/dia na TXAF; de 12,6 e 11,1 kg MS/100 $\mathrm{kg}$ PC/dia nas OFMST e OFMSL, respectivamente (Tabela 2).

Tabela 2. Taxa de acúmulo de forragem (TXAF), oferta de massa seca total (OFMST) e de lâminas (OMSL) em capim 'Mombaça' manejado sob diferentes intensidades de pastejo no semiárido baiano.

\begin{tabular}{|c|c|c|c|c|c|c|c|}
\hline \multirow{2}{*}{ Variáveis } & \multicolumn{4}{|c|}{ Altura de resíduo, $\mathrm{cm}$} & \multirow{2}{*}{ EPM* } & \multicolumn{2}{|c|}{$\mathrm{P}>$ Valor } \\
\hline & 10 & 25 & 40 & 55 & & Linear & Quadrático \\
\hline TXAF, kg MS/ha/dia & 82,5 & 149 & 303 & 450 & 5,36 & $0,000^{\mathrm{a}}$ & 0,000 \\
\hline OFMST, kg MS/100 kg PC/dia & 24,7 & 44,8 & 90,8 & 135 & 1,61 & $0,000^{\mathrm{b}}$ & 0,000 \\
\hline OFMSL, kg MS/100 kg PC/dia & 23,1 & 40,0 & 70,6 & 124 & 2,20 & $0,000^{\mathrm{c}}$ & 0,000 \\
\hline
\end{tabular}

*Erro padrão da média; PC, peso corporal. ${ }^{\mathrm{a}} \mathrm{y}=-26,117+8,3767 \mathrm{x} ;{ }^{\mathrm{b}} \mathrm{y}=-7,8367+2,5127 \mathrm{x} ;{ }^{\mathrm{c}} \mathrm{y}=-7,79+2,222 \mathrm{x}$

Segundo Flores et al. (2008), a menor taxa de acúmulo e oferta de massa seca em pastos manejados com maior intensidade de pastejo deve-se à maior remoção de folhas e da maior dependência de nitrogênio para sua rebrota. Semelhante ao presente estudo, Carvalho et al. (2017) registraram maior taxa de acúmulo de forragem em capim Mombaça manejado a uma altura de $50 \mathrm{~cm}$ de resíduo pós-pastejo. Porém, esse resultado pode estar associado à maior fração do caule, uma vez que, o manejo com um elevado resíduo permite alongamento do caule da planta. Euclides et al. (2016) constataram que a taxa de acúmulo de forragem e a massa de forragem pré-pastejo foram semelhantes nas alturas pós-pastejo de 30 e $50 \mathrm{~cm}$ em capim 'Mombaça' manejado em Campo Grande, Mato Grosso. Estes autores também verificaram que o capim Mombaça manejado a $50 \mathrm{~cm}$ de altura de resíduo favorece a seleção pelos animais.

As temperaturas médias ambientais e umidades relativas do ar nos meses de setembro a dezembro de 2019 estão apresentadas na Figura 1, onde se observa valores máximos variando de 30,7 a $32,9^{\circ} \mathrm{C}$ e 47,4 a 39,4\%, respectivamente. De acordo com Mac-Lean et al. (2011), em termos gerais, a zona de conforto térmico para bovinos europeus adultos é entre -1 e $16^{\circ} \mathrm{C}$ e para zebuínos adultos entre 10 e 27 ${ }^{\circ} \mathrm{C}$, com umidade relativa do ar variando de 60 a $70 \%$. Portanto, as variáveis climáticas poderiam explicar os resultados da temperatura de superfície de pelame (TSP) mensurado nas vacas (Tabela 3), em que se verificou aumento linear $(\mathrm{P}<0,05)$ de 32,2 para $36,4^{\circ} \mathrm{C}$, ao longo dos meses de avaliação. Antes do pastejo $(06: 00 \mathrm{~h})$, as vacas estavam em um ambiente sombreado, o que contribuiu para a menor TSP, que variou de 30,2 a $33,7^{\circ} \mathrm{C}$. Esse resultado demonstrou que, possivelmente, a ausência de árvores na área de pastagem, com consequente falta de sombreamento direto e indireto, contribuiu para a maior TSP dos animais durante os horários de pastejo. O estresse térmico em bovinos dificulta a dissipação de calor, e consequentemente aumenta a temperatura corpórea, podendo afetar o desempenho, devido à

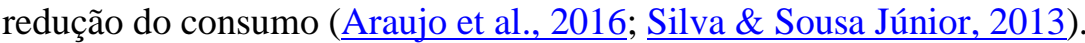

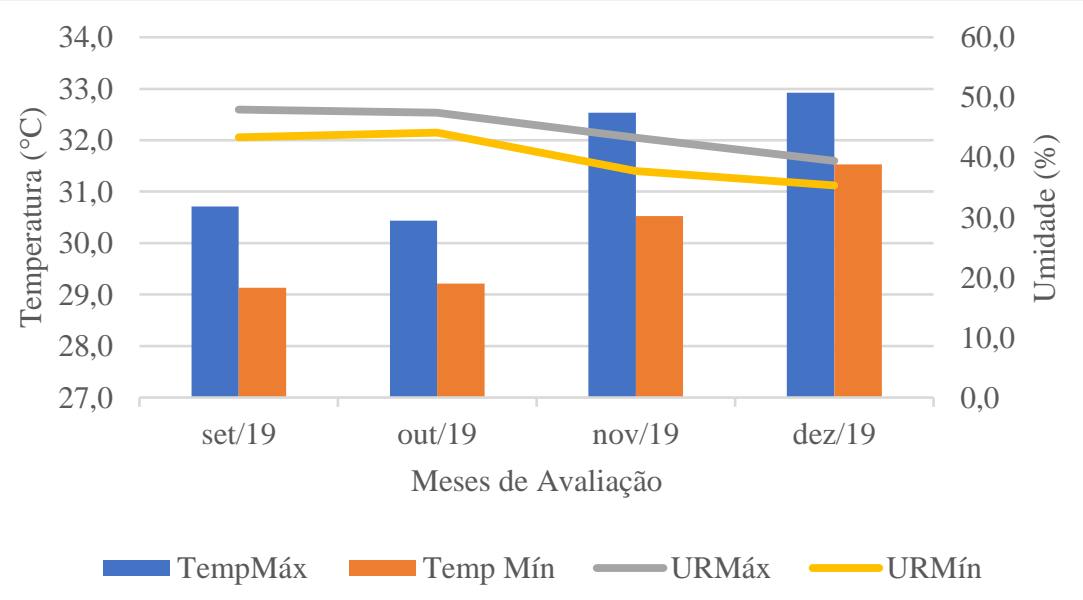

Figura 1. Temperaturas e umidades máximas e mínimas do ar durante os meses de setembro a dezembro de 2019, no município de Barra, semiárido baiano. Fonte: Adaptado de INMET (2019a). 
Nas condições climáticas da região semiárida do Oeste da Bahia, as altas temperaturas durante o período seco do ano poderiam propiciar desconforto térmico nos animais, uma vez que o ITU observado durante os meses de outubro a dezembro indicou estado de alerta, com média em torno de 72 a 78 , mas não chegou à zona de perigo, acima de 79, com exceção em um dia do mês de novembro (Figura 2). Souza et al. (2010) relataram que o ambiente físico sombreado apresenta uma redução em mais de 50,0\% da carga térmica radiante, sendo indispensável para bovinos manterem a homeotermia. Ainda, segundo esses autores, para bovinos de raças leiteiras, independente do estágio fisiológico ou categoria animal, é imprescindível a utilização de sombreamento para garantir o bem-estar, o conforto térmico e a maior produtividade. De acordo com o INMET (2019b), o ITU é um bom indicador da condição de desconforto térmico a que o animal está sujeito, sendo o recomendado para bovinos de no máximo 72. Acima de 72, caracteriza-se o início do estresse por calor, comprometendo as funções fisiológicas e o desempenho animal.

Tabela 3. Temperatura da superfície de pelame (TSP) e comportamento ingestivo de vacas mestiças sob diferentes intensidades de pastejo no semiárido baiano.

\begin{tabular}{|c|c|c|c|c|c|c|c|}
\hline \multirow{2}{*}{ Variáveis } & \multicolumn{4}{|c|}{ Altura de resíduo, $\mathrm{cm}$} & \multirow{2}{*}{$\mathrm{EPM}^{*}$} & \multicolumn{2}{|c|}{$\mathrm{P}>$ Valor } \\
\hline & 10 & 25 & 40 & 55 & & Linear & Quadrático \\
\hline TSP antes do pastejo, ${ }^{\circ} \mathrm{C}$ & 30,2 & 32,4 & 32,9 & 33,7 & 0,274 & $0,000^{\mathrm{a}}$ & 0,001 \\
\hline TSP durante o pastejo, ${ }^{\circ} \mathrm{C}$ & 32,2 & 35,2 & 36,1 & 36,4 & 0,309 & $0,000^{\mathrm{b}}$ & 0,000 \\
\hline Taxa de bocado, $\mathrm{n}^{\circ} /$ minuto & 1,05 & 1,29 & 1,80 & 2,34 & 0,049 & $0,000^{\mathrm{c}}$ & 0,000 \\
\hline Taxa de bocado, n $\%$ dia & 377 & 463 & 646 & 842 & 17,9 & $0,000^{\mathrm{d}}$ & 0,000 \\
\hline Estação alimentar, $\mathrm{n}$ /minuto & 2,34 & 1,58 & 1,22 & 0,84 & 0,057 & $0,000^{\mathrm{e}}$ & 0,000 \\
\hline Estação alimentar, $\mathrm{n}$ /dia & 842 & 569 & 441 & 303 & 20,5 & $0,000^{f}$ & 0,000 \\
\hline
\end{tabular}

*Erro padrão da média. ${ }^{\mathrm{a}} \mathrm{y}=29,917+0,0733 \mathrm{x} ;{ }^{\mathrm{b}} \mathrm{y}=32,05+0,09 \mathrm{x} ;{ }^{\mathrm{c}} \mathrm{y}=0,671+0,0292 \mathrm{x} ;{ }^{\mathrm{d}} \mathrm{y}=240,1+10,52 \mathrm{x} ;{ }^{\mathrm{e}} \mathrm{y}=2,548-$ $0,0324 x ;{ }^{f} y=916,83-11,633 x$.

A maioria dos dados sobre ITU referem-se a regiões temperadas e para animais especializados na produção de leite e menos tolerantes ao calor. Dessa forma, deve-se ter cautela ao se analisar o ITU em zonas tropicais, pois os valores críticos podem estar superestimados e fornecer uma interpretação incorreta do ambiente térmico para animais que já são adaptados nesse ambiente (Brettas et al., 2019). Azevedo et al. (2005), estudando o ITU de vacas leiteiras mestiças (Holandês-Zebu) em lactação, relataram que o grau de sangue dos animais influencia o nível crítico de ITU; sendo que, os valores críticos superiores de ITU para os animais dos grupos genéticos 1/2,3/4 e 7/8 HZ são de 79, 77 e 76, respectivamente. O tempo de exposição à radiação solar também é um fator importante para se analisar o ITU, segundo (Dias et al., 2012). Estes autores observaram que, no Piauí, em vacas mestiças (Holandês-Gir) expostas a duas horas de radiação solar, entre as 10:00 e 12:00 horas, o ITU foi considerado crítico, causando estresse térmico e afetando o desempenho, diferente de vacas expostas a uma hora de radiação solar, das 10:00 às 11:00 horas, as quais não sofreram expressivas alterações fisiológicas.

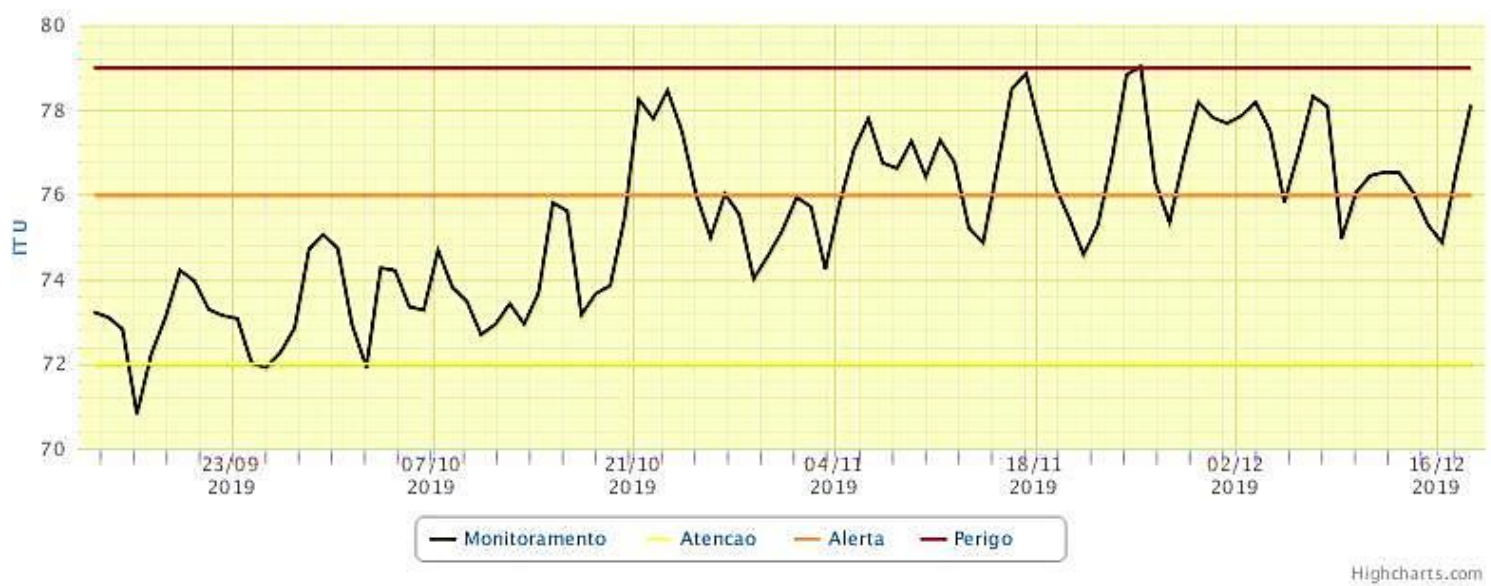

Figura 2. Índice de Temperatura e Umidade (ITU) durante os meses de setembro a dezembro de 2019 no município de Barra, semiárido baiano. Fonte: INMET $(\underline{2019 b})$.

Para o comportamento ingestivo dos animais, verificou-se aumento linear $(\mathrm{P}<0,05)$ nas taxas de bocado com o aumento das alturas de resíduo pós-pastejo, em que, para cada aumento de $5,0 \mathrm{~cm}$ na altura de resíduo, espera-se um aumento de $0,146 \mathrm{n} \%$ minuto e 52,6 n\%dia, respectivamente (Tabela 3). 
Observou-se que a taxa de bocados foi influenciada pela intensidade de pastejo, ou seja, com o aumento do resíduo de forragem, permitiu-se maior disponibilidade de massa seca para apreensão durante o pastejo. Resultado semelhante foi registrado por Melo et al. (2016), que observaram aumento no número de bocados por estação alimentar em intensidades de pastejo de 30, 40 e $50 \mathrm{~cm}$ em pasto de capim 'BRS Piatã'. Flores et al. (2008) trabalhando com dois cultivares de Brachiaria brizantha (Marandu e Xaraés) observaram que nos pastos manejados a $15 \mathrm{~cm}$, os animais tiveram maior dificuldade de seleção e de preensão da forragem, em razão da menor oferta de lâminas foliares e maior quantidade de material morto. Com o aumento na altura do resíduo para $40,0 \mathrm{~cm}$, a dificuldade encontrada pelos animais deveuse a maior quantidade de colmos presente na forragem.

$\mathrm{O}$ deslocamento pelas estações alimentares reduziu linearmente $(\mathrm{P}<0,05)$, em que, para cada aumento de $5,0 \mathrm{~cm}$ na altura de resíduo, espera-se uma redução de $0,162 \mathrm{n} \%$ minuto e $58,2 \mathrm{n} /$ dia, respectivamente (Tabela 3). Quando a oferta de forragem aumenta observa-se maior permanência na estação alimentar, devido a maior quantidade de massa no bocado, o que pode não incentivar os animais a trocar de estações, como nas alturas de resíduo de 40,0 e 55,0 cm (Tabela 3). Segundo Teixeira et al. (2011), em situações limitantes de forragem, como no pasto manejado a 10,0 e 25,0 cm de altura de resíduo (Tabela 3), os animais apreendem pouca massa a cada bocado e tendem a apresentar maiores frequências de deslocamentos, curtos e retilíneos, na busca de melhores condições de consumo. Resultado semelhante foi encontrado por Gregorini et al. (2011), com aumento dos deslocamentos entre as estações alimentares por bovinos e redução dos bocados, em alturas de resíduos mais baixas. Palhano et al. (2006) observaram que, com o aumento na altura do dossel, o número de estações alimentares por minuto foi menor, devido a maior disponibilidade de forragem.

Devido ao incremento na OFMST, verificou-se aumento linear $(\mathrm{P}<0,05)$ no consumo de matéria seca (CMS) e ganho médio diário (GMD) (Tabela 4). Segundo o NRC (2001), o consumo médio de MS em uma vaca leiteira de aproximadamente $500 \mathrm{~kg}$ é de cerca de $2,8 \mathrm{~kg}$ MS/100 kg PC. Dessa forma, os resultados obtidos no presente estudo indicaram que em todas as intensidades de pastejo avaliadas a OFMST seria suficiente para manter uma vaca lactante de $500 \mathrm{~kg}$ durante um dia. Considerando-se que os animais em pastejo selecionam a forragem, a intensidade de pastejo com altura de resíduo de $40,0 \mathrm{~cm}$ seria a ideal, pois permitiria maior consumo voluntário e até aumento do número de animais por piquete, permitindo-se maior produção por área. Contudo, para se realizar a apreensão de forragem em uma pastagem abaixo da altura recomendada, ocorre gasto energético por parte dos animais, acarretando redução no desempenho (Barbosa et al., 2013). Isso explicaria o menor ganho de peso $(0,073 \mathrm{~kg} / \mathrm{dia})$ nos animais mantidos em intensidade de pastejo de $10,0 \mathrm{~cm}$, o que está associado a uma menor oferta de massa seca total e de lâminas foliares (Tabela 4). Segundo Brâncio et al. (2003), à medida que os animais selecionam as partes mais palatáveis das plantas, a pastagem apresenta proporção crescente de material não preferido ou recusado, como colmos e material morto, dificultando cada vez mais a seleção e a ingestão de forragem. Euclides et al. (2018) relataram menor GMD em animais pastejando capim 'Mombaça' com 30,0 cm de altura de resíduo, devido à menor razão folha:colmo e valor nutritivo, sendo considerado ideal $50,0 \mathrm{~cm}$ associado a uma altura de pré-pastejo de $90 \mathrm{~cm}$.

Tabela 4. Consumo de matéria seca (CMS) e escore de condição corporal (ECC) de vacas mestiças sob diferentes intensidades de pastejo em capim 'Mombaça' no semiárido baiano.

\begin{tabular}{|c|c|c|c|c|c|c|c|}
\hline \multirow{2}{*}{ Variável } & \multicolumn{4}{|c|}{ Altura de resíduo, $\mathrm{cm}$} & \multirow{2}{*}{ EPM* } & \multicolumn{2}{|c|}{$\mathrm{P}>$ Valor } \\
\hline & 10 & 25 & 40 & 55 & & Linear & Quadrático \\
\hline CMS, kg/dia & 12,4 & 13,8 & 16,3 & 17,3 & 0,076 & $0,000^{\mathrm{a}}$ & 0,000 \\
\hline CMS, \%PC & 2,47 & 2,68 & 3,09 & 3,19 & 0,016 & $0,000^{b}$ & 0,000 \\
\hline $\mathrm{PC}, \mathrm{kg}$ & 503 & 514 & 525 & 540 & - & - & - \\
\hline GMD, kg/dia & 0,073 & 0,347 & 0,392 & 0,495 & 0,040 & 0,002 & $0,005^{\mathrm{c}}$ \\
\hline ECC Vetscore & Verde & Verde & Verde & Verde & - & - & - \\
\hline ECC Visual & 3,00 & 3,00 & 3,25 & 3,50 & - & - & - \\
\hline
\end{tabular}

*Erro padrão da média; PC, peso corporal; GMD, ganho médio diário. ${ }^{\mathrm{a}} \mathrm{y}=11,223+0,1147 \mathrm{x} ;{ }^{\mathrm{b}} \mathrm{y}=2,3007+0,0171 \mathrm{x} ;{ }^{\mathrm{c}} \mathrm{y}=$ $0,0427+0,0087 x$

Além do GMD, a avaliação do ECC é um bom indicativo do desempenho animal. Entre as técnicas disponíveis para se aferir o ECC, o Vetscore ${ }^{\circledR}$ é considerado um dispositivo simples, barato, facilmente disponível e que não consome tempo para se determinar a condição corporal dos animais (Pfeifer et al., 2017). No presente estudo, o ECC avaliado pelo Vetscore ${ }^{\circledR}$ foi semelhante nas diferentes intensidades 
de pastejo, com registro da coloração verde, que equivale ao ECC variando de 2,75 a 4,50 segundo Pfeifer et al. (2017), o que corrobora com o registrado por meio tátil e visual (Tabela 1). Todavia, entre as alturas de 40 e $55 \mathrm{~cm}$ de resíduo, foi observado um incremento no ECC avaliado por meio visual (Tabela 4), que poderia ser comprovado pela variação do peso corporal e GMD dos animais. Esse resultado está provavelmente relacionado a uma maior oferta de forragem para o pastejo, evitando gasto energético por parte dos animais e consequentemente redução das reservas corporais armazenadas (Barbosa et al., 2013).

Conclui-se que as características produtivas do capim 'Mombaça' e o comportamento ingestivo de animais em pastejo são afetados pela intensidade de pastejo. Portanto, recomenda-se a intensidade de pastejo de 45,2 cm (altura de resíduo), por permitir aliar adequada produção de massa seca e oferta de lâminas foliares, e menor proporção de colmos e material morto. Além disso, permite-se maior quantidade de biomassa de pasto à disposição dos animais, comprovado pela maior quantidade de bocados e menor deslocamento entre estações alimentares, aliado a um consumo e desempenho adequados nas vacas mestiças de aptidão leiteira criadas no semiárido do Oeste da Bahia.

\section{Referências}

Alexandrino, E., Cândido, M. J. D., \& Gomide, J. A. (2011). Fluxo de biomassa e taxa de acúmulo de forragem em capim Mombaça mantido sob diferentes alturas. Revista Brasileira de Saúde e Produção Animal, 12(1), 59-71.

Andrade, P. X., Ferreira, M. A., Azevedo, M., Silva, E. C., Urbano, S. A., Conceição, M. G., \& Silva, J. L. (2017). Feed handling of lactating crossbred cows maintained in a semi-arid region during the hot season: physiological parameters, ingestive behavior and performance. Animal Science Journal, 88(1), 166-172. DOI: https://doi.org/10.1111/asj.12618

Araujo, J. I. M., Araujo, A. C., Fonseca, W. J. L., Barros Junior, C. P., Luz, C. S. M., Araujo, J. M., Farias, L. A., \& Sousa Júnior, S. C. (2016). Efeitos das variáveis climáticas sobre características fisiológicas de vacas mestiças (Holandês x Gir) em lactação. Revista Acadêmica: Ciência Animal, 14, 185-193. DOI: https://doi.org/10.7213/academica.14.2016.20

Azevedo, M., Pires, M. de F. Á., Saturnino, H. M., Lana, Â. M. Q., Sampaio, I. B. M., Monteiro, J. B. N., \& Morato, L. E. (2005). Estimation of upper critical levels of the temperature-humidity index for 1/2, 3/4 e 7/8 lactating Holstein-Zebu dairy cows. Revista Brasileira de Zootecnia, 34(6), 2000-2008. DOI: http://dx.doi.org/10.1590/S1516-35982005000600025

Barbosa, M. A. A. F., Castro, L. M., Barbero, R. P., Brito, V. C., Miorin, R. L., Saad, R. M., Ribeiro, E. L. A., \& Bumbieris Junior, V. H. (2013). Comportamento ingestivo de bovinos mantidos em pastos de Brachiaria brizantha cv. Xaraés manejado em diferentes alturas de pastejo. Semina-Ciencias Agrarias, 6, 4113-4120. DOI: https://doi.org/10.5433/1679-0359.2013v34n6Supl2p4145

Brâncio, P. A., Nascimento Júnior, D., Euclides, V. P. B., Fonseca, D. M., Almeida, R. G., Macedo, M. C. M., \& Barbosa, R. A. (2003). Avaliação de três cultivares de Panicum maximum Jacq. sob pastejo: composição da dieta, consumo de matéria seca e ganho de peso animal. Revista Brasileira de Zootecnia, 32(5), 1037-1044. DOI: http://dx.doi.org/10.1590/S1516-35982003000500002

Brettas, P. K. M., Guimarães, E. C., \& Nascimento, M. R. B. M. (2019). Dados de estação meteorológica oficial subestimam o estresse por calor em bovinos leiteiros criados em ambiente tropical. Arquivo Brasileiro de Medicina Veterinária e Zootecnia, 71(3), 967-976. DOI: https://doi.org/10.1590/1678$\underline{4162-10432}$

Carnevalli, R. A., Silva, S. C., Bueno, A. A., Uebele, M. C., Bueno, F. O., Hodgson, J., Silva, G. N., \& Morais, J. P. G. (2006). Herbage production and grazing losses in Panicum maximum cv. Mombaça under four grazing managements. Tropical Grasslands, 40(3), 165.

Carvalho, A. L. S., Martuscello, J. A., Almeida, O. G., Braz, T. G. S., Cunha, D. de N. F. V., \& Jank, L. (2017). Production and quality of Mombaça grass forage under different residual heights. Acta Scientiarum. Animal Sciences, 39(2), 143-148. DOI: https://doi.org/10.4025/actascianimsci.v39i2.34599 
Detmann, E., Souza, M. A., Valadares Filho, S. C., Queiroz, A. C., Berchielli, T. T., Saliba, E. O. S., Cabral, L. S., Pina, D. S., Ladeira, M. M., \& Azevedo, J. A. G. (2012). Métodos para análise de alimentos. Suprema.

Dias, T. P., Sousa Júnior, S. C., Oliveira, R. G., \& Santos, K. R. (2012). Efeito da exposição à radiação solar sobre parâmetros fisiológicos e estimativa do declínio na produção de leite de vacas mestiças (Holandês X Gir) no sul do estado do Piauí. Comunicata Scientiae, 3(4), 299-305.

Euclides, V. P. B, Carpejani, G. C., Montagner, D. B., Nascimento Junior, D., Barbosa, R. A., \& Difante, G. S. (2018). Maintaining post-grazing sward height of Panicum maximum (cv. Mombaça) at $50 \mathrm{~cm}$ led to higher animal performance compared with post-grazing height of $30 \mathrm{~cm}$. Grass and Forage Science, 73(1), 174-182. DOI: https://doi.org/10.1111/gfs.12292

Euclides, Valéria Pacheco Batista, Lopes, F. C., Nascimento Júnior, D., Silva, S. C., Difante, G. S., \& Barbosa, R. A. (2016). Steer performance on Panicum maximum (cv. Mombaça) pastures under two grazing intensities. Animal Production Science, 56(11), 1849-1856. DOI: https://doi.org/10.1071/AN14721

Ferguson, J., Byers, D., Ferry, J., Johnson, P., Ruegg, P., \& Weaver, L. (1994). Body condition of lactating cows. Agri-Cultural Ractice, 15, 17-21.

Flores, R. S., Euclides, V. P. B., Abrão, M. P. C., Galbeiro, S., Difante, G. S., \& Barbosa, R. A. (2008). Desempenho animal, produção de forragem e características estruturais dos capins marandu e xaraés submetidos a intensidades de pastejo. Revista Brasileira de Zootecnia, 37(8), 1355-1365. DOI: https://doi.org/10.1590/S1516-35982008000800004

Gregorini, P., Gunter, S. A., Bowman, M. T., Caldwell, J. D., Masino, C. A., Coblentz, W. K., \& Beck, P. A. (2011). Effect of herbage depletion on short-term foraging dynamics and diet quality of steers grazing wheat pastures. Journal of Animal Science, 89(11), 3824-3830. DOI: https://doi.org/10.2527/jas.2010-3725

Instituto Nacional de Meteorologia - INMET. $\left(2019^{\mathrm{a}}\right)$. Estações Automáticas. Acesso em: dezembro de 2019. Disponível em: http://www.inmet.gov.br/portal/index.php?r=estacoes/estacoesAutomaticas.

Instituto Nacional de Meteorologia - INMET. (2019b). SISDAGRO - Conforto térmico bovino. Acesso em: dezembro de 2019.2 Disponível em: http://sisdagro.inmet.gov.br/sisdagro/app/climatologia/confortotermicobovino.

Jamieson, W. S., \& Hodgson, J. (1979). The effects of variation in sward characteristics upon the ingestive behaviour and herbage intake of calves and lambs under a continuous stocking management. Grass and Forage Science, 34(4), 273-282. DOI: https://doi.org/10.1111/j.13652494.1979.tb01479.x

Mac-Lean, P. A. B., Barbosa, O. R., Jobim, C. C., Gasparino, E., Santos, G. T., \& Faria, L. A. N. (2011). Sombra artificial e método de fornecimento de concentrado no comportamento e desempenho de bezerros desmamados. Acta Scienciarum.Animal Sciences, 33(4), 409-415. DOI: https://doi.org/10.4025/actascianimsci.v33i4.10672

Melo, J. C., Alexandrino, E., Paula Neto, J. J. de, Rezende, J. M. de, Silva, A. A. M., Silva, D. V., \& Oliveira, A. K. R. (2016). Comportamento ingestivo de bovinos em capim-Piatã sob lotação intermitente em resposta a distintas alturas de entrada. Revista Brasileira de Saúde e Produção Animal, 17(3), 385-400. DOI: https://doi.org/10.1590/S1519-99402016000300006

Minson, D. (2012). Forage in ruminant nutrition (Vol. 1). Academic Press. DOI: https://doi.org/10.1016/b978-0-12-498310-6.50007-9

Morais, L. F., Carvalho, C. A. B., Assis Anjos, A. N., Viegas, C. R., \& Silva, P. H. F. (2018). Avanços na avaliação de pastagens cultivadas com forrageiras tropicais no Brasil: Uma Revisão. Brazilian Journal of Applied Technology for Agricultural Science, 11(2), 125-136.

NRC. (2001). Nutrient Requirements of Dairy Cattle (7th (ed.); 7th rev.). Natl. Acad. Press, Washington, DC.

Palhano, A. L., Carvalho, P. C. F., Dittrich, J. R., Moraes, A., Silva, S. C., \& Monteiro, A. L. G. (2006). Padrões de deslocamento e procura por forragem de novilhas leiteiras em pastagem de capimmombaça. Revista Brasileira de Zootecnia, 35(6), 2253-2259. DOI: https://doi.org/10.1590/s151635982006000800008 
Pfeifer, L. F. M., Castro, N. A., Neves, P. M. A., Cestaro, J. P., \& Siqueira, L. G. B. (2017). Development and validation of an objective method for the assessment of body condition scores and selection of beef cows for timed artificial insemination. Livestock Science, 197, 82-87. DOI: https://doi.org/10.1016/j.livsci.2017.01.011

Reis, R. A., Ruggieri, A. C., Casagrande, D. R., \& Páscoa, A. G. (2009). Suplementação da dieta de bovinos de corte como estratégia do manejo das pastagens. Revista Brasileira de Zootecnia, 38, 147 159. DOI: https://doi.org/10.1590/s1516-35982009001300016

Ribeiro Filho, H. M. N., Setelich, E. A., Crestani, S., Dias, K. M., Mantovani, C., \& Valenti, J. (2011). Inter-relação entre o tempo de pastejo diurno e o consumo de forragem em vacas leiteiras em pastejo rotativo. Ciência Rural, 41(11), 2010-2013. DOI: https://doi.org/10.1590/S010384782011005000140

Rocha, W. J. B., Silva, R. R., Silva, F. F., Carvalho, G. G. P., Silva, A. P. G., Silva, J. W. D., Paixão, T. R., Freitas, T. B., Mendes, F. B. L., \& Barroso, D. S. (2019). Intake, digestibility, and growth performance of Girolando bulls supplemented on pasture in Bahia, Brazil. Tropical Animal Health and Production, 1-8. DOI: https://doi.org/10.1007/s11250-019-01824-2

Silva, É. C. L., Modesto, E. C., Azevedo, M., Andrade Ferreira, M., Dubeux Júnior, J. C. B., \& Schuler, A. R. P. (2009). Efeitos da disponibilidade de sombra sobre o desempenho, atividades comportamentais e parâmetros fisiológicos de vacas da raça Pitangueiras. Acta Scientiarum. Animal Sciences, 31(3). DOI: http://dx.doi.org/10.4025/actascianimsci.v31i3.5279

Silva, S. C., Bueno, A. A. de O., Carnevalli, R. A., Uebele, M. C., Bueno, F. O., Hodgson, J., Matthew, C., Arnold, G. C., \& Morais, J. P. G. (2009). Sward structural characteristics and herbage accumulation of Panicum maximum cv. Mombaça subjected to rotational stocking managements. Scientia Agricola, 66(1), 8-19. DOI: https://doi.org/10.1590/s0103-90162009000100002

Silva, T. P. D., \& Sousa Júnior, S. C. (2013). Produção de leite de vacas submetidas a diferentes períodos de exposição à radiação solar no sul do Piauí. Revista Agrarian, 6(21), 320-325.

Sollenberger, L. E., \& Cherney, D. J. R. (1995). Evaluating forage production and quality. Forages: The Science of Grassland Agriculture, 2, 97-110.

Souza, A. N. M., Rocha, M. G., Pötter, L., Roso, D., Glienke, C. L., \& Oliveira Neto, R. A. (2011). Comportamento ingestivo de novilhas de corte em pastagem de gramíneas anuais de estação quente. Revista Brasileira de Zootecnia, 40(8), 1662-1670. DOI: https://doi.org/10.1590/S1516$\underline{35982011000800006}$

Souza, B. B., Silva, I. J. O., Mellace, E. M., Santos, R. F. S., Zotti, C. A., \& Garcia, P. R. (2010). Avaliação do ambiente físico promovido pelo sombreamento sobre o processo termorregulatório em novilhas leiteiras. Agropecuária Científica Seminário, 6(2), 59-65. DOI: https://doi.org/10.30969/acsa.v6i2.69

Teixeira, F. A., Bonomo, P., Pires, A. J. V., Silva, F. F., Rosa, R. C. C., \& Nascimento, P. V. N. (2011). Diferimento de pastos de Brachiaria decumbens adubados com nitrogênio no início e no final do período das águas. Revista Brasileira de Zootecnia, 40(7), 1480-1488. DOI: https://doi.org/10.1590/s1516-35982011000700012.

Histórico do artigo:

Recebido: 10 de setembro de 2020

Licenciamento: Este artigoé publicado na modalidade Acesso Aberto sob a licença Creative Commons Atribuição 4.0 (CC-BY 4.0), a qual permite uso irrestrito, distribuição, reprodução em qualquer meio, Disponível online: 12 de janeiro de 2021. desde que $\mathrm{o}$ autor $\mathrm{a}$ a fonte sejam devidamente creditados. 